\title{
Stochastic programming technique for portfolio optimization with minimax risk and bounded parameters
}

\author{
P KUMAR $^{1}$, G PANDA $^{2, *}$ and U C GUPTA ${ }^{2}$ \\ ${ }^{1}$ Department of Mathematics, SRM Institute of Science and Technology, Chennai, India \\ ${ }^{2}$ Department of Mathematics, Indian Institute of Technology, Kharagpur, India \\ e-mail: geetanjali@maths.iitkgp.ernet.in
}

MS received 24 August 2017; revised 11 January 2018; accepted 18 January 2018; published online 24 July 2018

\begin{abstract}
In this paper a portfolio optimization problem with bounded parameters is proposed taking into consideration the minimax risk measure, in which liquidity of the stocks is allied with selection of the portfolio. Interval uncertainty of the model is dealt with through a fusion between interval and random variable. As a result of this, the interval inequalities are converted to chance constraints. A solution methodology is developed using this concept to obtain an efficient portfolio. The theoretical developments are illustrated on a large data set taken from National Stock Exchange, India.
\end{abstract}

Keywords. Stochastic programming; minimax risk; portfolio optimization; interval analysis.

\section{Introduction}

Basic criteria of a general portfolio selection problem take care of an investment strategy to fetch the maximum possible return with minimum risk. Starting from Markowitz [1, 2], several authors have reformulated portfolio optimization model accepting various factors of the financial market such as transaction cost, market impact cost, liquidity, short selling, etc. Some recent references with risk measure as standard deviation are [3-10]. The risk functions such as semi-variance [2, 11], mean absolute deviation [12, 13], skewness [14, 15], down-side risk [16-19], etc. are also considered in place of variance risk. The minimax risk function, introduced by Young [20], minimizes the maximum loss over all past observation periods, for a given level of return. The special structure of minimax risk function is $\ell_{\infty}$-risk measure, which was proposed by Cai et al [21] and further improved by Teo and Yang [22] and Deng et al [23]. Wu et al [24] extended the result of Deng et al [23] including the short sale restriction. Recently, Sharma and Mehra [25] developed a portfolio optimization model with minimax measure as safety constraint, which enables a trade-off between return and risk with a fixed safety value. Portfolio selection of this paper is based on minimax risk measure.

Due to the presence of uncertainty in the financial market, some parameters like risk, return, etc., cannot be estimated directly. These parameters are generally estimated using probability theory, creditability theory, etc. However, sometimes it is difficult to identify the proper distribution

*For correspondence function for these uncertain parameters. In such cases an investor can easily find the lower and upper bound of these parameters from the historical data and consider the parameters as closed intervals. In recent years this idea has been accepted by Ida [26], Lai et al [27], Giove et al [28], Liu [29] and Tan [30]. Liu et al [31] proposed a portfolio optimization model for multiple time periods considering the return, risk and liquidity in the form of closed intervals with certain degree of diversification. Wu et al [32] proposed an interval portfolio selection model in which both the returns and the risks of the assets are considered as intervals, and obtained a non-inferior solution that improves and generalizes the Markowitz's MV model. A portfolio rebalancing model is studied by Kumar et al [33] with return, risk, fixed transaction cost and variable transaction cost as closed intervals, which can be applied to obtain optimal investment strategy for multiple time horizons. An extension of Lai et al [27]'s model is discussed in Kumar et al [34-36] considering proportion of total investment of each stock in terms of closed intervals.

In this paper a portfolio optimization model is studied with $\ell_{\infty}$-risk function accepting the parameters of the model as closed intervals. Methodology of this paper is different from the afore-mentioned existing methodologies, where interval parameters are dealt with by scalarizing the portfolio model using only the lower and upper bounds of the intervals. By this scalarization process some times there may be a chance of loosing the information between the bounds of the intervals, which are involved in the process. To address this aspect, we have developed a concept of fusion between an interval and a normally distributed 
random variable using $3 \sigma$ rule. This process has two advantages:

- Loosing information between bounds can be avoided when the parameters are considered as intervals,

- Botheration for the selection of suitable distribution function to estimate the uncertain parameters can be avoided when the parameters are considered in probabilistic sense instead of intervals.

Based on this concept, a solution methodology is developed in this paper to obtain an efficient portfolio. The methodology is compared to two existing methodologies on a large data set. Remaining part of the paper is organized as follows. Preliminaries on interval analysis are provided in section 2 . Section 3 discusses the model formulation of problem and solution methodology, which is summarized in a step by step procedure. Further, to show the applicability of model for large data sets, a real life example is considered in section 4 for the data taken from National Stock Exchange, India. Results are compared to some existing results.

\section{Preliminaries}

Following notations are used throughout this paper. $\mathbf{I}(\mathfrak{R})=$ set of closed intervals. $\overline{\mathbf{A}} \in \mathbf{I}(\mathfrak{R})$ is a set, $\overline{\mathbf{A}}=\left[a^{L}, a^{U}\right]=\left\{x \in \mathfrak{R} \mid a^{L} \leq x \leq a^{U}\right\} . \overline{\mathbf{A}}$ is said to be a degenerate interval if $a^{L}=a^{R} . \overline{\mathbf{A}} \geq 0$ means every element of $\overline{\mathbf{A}}$ is non-negative, that is, $a^{L} \geq 0$. An interval can also be represented in terms of its mean and spread as $\langle m(\overline{\mathbf{A}}), w(\overline{\mathbf{A}})\rangle$, where $m(\overline{\mathbf{A}})=\frac{a^{L}+a^{U}}{2}$ and $w(\overline{\mathbf{A}})=\frac{a^{U}-a^{L}}{2}$. Throughout this paper an interval is represented as bold capital letter with upper bar.

- Algebraic operations in $\mathbf{I}(\mathfrak{R})$ are defined as follows. For $\overline{\mathbf{A}}, \overline{\mathbf{B}} \in \mathbf{I}(\mathfrak{R}), c \in \mathfrak{R}$ and $* \in\{+,-, \times, \div\}$,

$$
\begin{aligned}
\overline{\mathbf{A}} \circledast \overline{\mathbf{B}} & =\{a * b \mid a \in \overline{\mathbf{A}}, b \in \overline{\mathbf{B}}\} \\
& =\left[\min _{a \in \overline{\mathbf{A}}, b \in \overline{\mathbf{B}}}(a * b), \max _{a \in \overline{\mathbf{A}}, b \in \overline{\mathbf{B}}}(a * b)\right],
\end{aligned}
$$

in the case of division, $0 \notin \overline{\mathbf{B}}$.

$$
\begin{gathered}
c \cdot \overline{\mathbf{A}}=\left[c a^{L}, c a^{U}\right], \text { if } c \geq 0 ; \\
{\left[c a^{U}, c a^{L}\right], \text { if } c \leq 0}
\end{gathered}
$$

for all $\overline{\mathbf{B}} \in \mathbf{I}(\mathfrak{R})$.

- $\overline{\mathbf{A}} \ominus \overline{\mathbf{A}} \neq[0,0]$.

To address this issue, we use Markov [37] subtraction, which is a nonstandard difference $\ominus_{M}$, as follows:

$$
\overline{\mathbf{A}} \ominus_{M} \overline{\mathbf{B}}= \begin{cases}{\left[a^{L}-b^{L}, a^{U}-b^{U}\right],} & \text { if } w(\overline{\mathbf{A}}) \geq w(\overline{\mathbf{B}}) \\ {\left[a^{U}-b^{U}, a^{L}-b^{L}\right],} & \text { if } w(\overline{\mathbf{A}})<w(\overline{\mathbf{B}})\end{cases}
$$

and $\overline{\mathbf{A}} \ominus_{M} \overline{\mathbf{A}}=[0,0] \triangleq \overline{\mathbf{0}}$.
- Denote $\overline{\mathbf{A}}_{1} \oplus \overline{\mathbf{A}}_{2} \oplus \ldots \oplus \overline{\mathbf{A}}_{n} \triangleq \sum_{j=1}^{n} \overline{\mathbf{A}}_{j}$

- For two intervals $\overline{\mathbf{A}}=\langle m(\overline{\mathbf{A}}), w(\overline{\mathbf{A}})\rangle \quad$ and $\overline{\mathbf{B}}=\langle m(\overline{\mathbf{B}}), w(\overline{\mathbf{B}})\rangle$,

$$
\left.\begin{array}{cll}
m(\overline{\mathbf{A}} \oplus \overline{\mathbf{B}}) & =m(\overline{\mathbf{A}})+m(\overline{\mathbf{B}}) \\
m\left(\overline{\mathbf{A}} \ominus_{M} \overline{\mathbf{B}}\right) & =m(\overline{\mathbf{A}})-m(\overline{\mathbf{B}}) \\
w(\overline{\mathbf{A}} \oplus \overline{\mathbf{B}}) & =w(\overline{\mathbf{A}})+w(\overline{\mathbf{B}}) . \\
w\left(\overline{\mathbf{A}} \ominus_{M} \overline{\mathbf{B}}\right) & =|w(\overline{\mathbf{A}})-w(\overline{\mathbf{B}})|
\end{array}\right\}
$$

- $\mathbf{I}(\mathfrak{R})$ is not a totally ordered set and several partial order relations exist in $\mathbf{I}(\mathfrak{R})$. The most common partial order relation ( $)$ is: for $\overline{\mathbf{A}}, \overline{\mathbf{B}} \in \mathbf{I}(\mathfrak{R})$,

$$
\begin{aligned}
& \overline{\mathbf{A}} \preceq \overline{\mathbf{B}} \text { iff } a^{L} \leq b^{L} \text { and } a^{U} \leq b^{U} \\
& \overline{\mathbf{A}} \prec \overline{\mathbf{B}} \text { iff } \overline{\mathbf{A}} \preceq \overline{\mathbf{B}} \text { and } \overline{\mathbf{A}} \neq \overline{\mathbf{B}} .
\end{aligned}
$$

\section{Portfolio selection model with $l_{\infty}$ risk measure}

As discussed earlier in this paper, we consider portfolio selection problem with interval parameters and $\ell_{\infty}$ risk function due to Cai et al [21]. Following assumptions and notations are used throughout the paper to formulate the model.

$n \quad$ total number of risky securities

$\mathrm{R}_{j} \quad$ random rate of return of $j$ th security,

$j=1,2, \ldots, n$.

rate of return of $j$ th security at time $t$,

$j=1,2, \ldots, n, t=1,2, \ldots, T$

expected rate of return of $j$ th security, $\mu_{j}=$ $\mathrm{E}\left(\mathrm{R}_{j}\right)=\frac{1}{T} \sum_{t=1}^{T} r_{j t}$

$\mu_{j}^{L}\left(\mu_{j}^{U}\right) \quad$ lower (upper) bound of the expected return of $j$ th security, estimated from historical data such that $\mu_{j}^{L} \leq \mu_{j} \leq \mu_{j}^{U}$, i.e., $\mu_{j} \in\left[\mu_{j}^{L}, \mu_{j}^{U}\right] \triangleq \overline{\boldsymbol{\mu}}_{j}$

$\mathrm{R} \quad$ random rate of return (time independent) per period on the total investment expected return (time independent) per period on the total investment, $\mu=\mathrm{E}(\mathrm{R})$

$\overline{\boldsymbol{\mu}} \quad$ interval that contains the expected return of investment, i.e., $\mu^{L} \leq \mu \leq \mu^{U}, \overline{\boldsymbol{\mu}}=\left[\mu^{L}, \mu^{U}\right]$.

$x_{j} \quad$ proportion of total investment on $j$ th security, $j=1,2, \ldots, n, \sum_{j=1}^{n} x_{j}=1$.

$q_{j} \quad$ expected absolute deviation of $\mathrm{R}_{j}$ from mean of $j$ th security, $q_{j} \triangleq \mathrm{E}\left(\left|\mathrm{R}_{j}-\mu_{j}\right|\right)$

Definition 3.1 [21] The $\ell_{\infty}$ risk function is defined as

$$
w_{\infty}(\mathbf{x})=\max _{1 \leq j \leq n}\left\{\mathrm{E}\left(\left|\mathrm{R}_{j}-\mu_{j}\right|\right) x_{j}\right\}=\max _{1 \leq j \leq n} q_{j} x_{j}
$$

where $q_{j}=\mathrm{E}\left(\left|\mathrm{R}_{j}-\mu_{j}\right|\right)=\frac{1}{T} \sum_{t=1}^{T}\left|r_{j t}-\mu_{j}\right|$. 


\subsection{Model formulation}

Expected return of a portfolio $\mathbf{x}=\left(x_{1}, x_{2}, \ldots, x_{n}\right)$ is $\mu(\mathbf{x})=\sum_{j=1}^{n} \mu_{j} x_{j}$. Since $\mu_{j} \in\left[\mu_{j}^{L}, \mu_{j}^{U}\right]$, the expected return of the portfolio $\mathbf{x}$ also varies in a closed interval, which can be represented mathematically as

$$
\overline{\boldsymbol{\mu}}(\mathbf{x})=\sum_{j=1}^{n}\left[\mu_{j}^{L}, \mu_{j}^{U}\right] x_{j} .
$$

Liquidity is one of the important factors that affects the future expected return of the portfolio. It is measured as the degree of probability of being able to convert an investment into cash without any significant loss in value. To measure the liquidity of a portfolio, turnover rate of the securities is used, which is the proportion of turnover volume to tradable volume of the security (see Fang et al [5], Yan [38]). The turnover rate $\left(l_{j}\right)$ of a security is random and the liquidity of money invested in any stock $l_{j} x_{j}$ with expected liquidity of the portfolio is $\mathrm{E}\left(\sum_{j=1}^{n} l_{j} x_{j}\right)=\sum_{j=1}^{n} \mathrm{E}\left(l_{j}\right) x_{j}$. As the future turnover rate of any security cannot be accurately predicted due to the presence of uncertainty factors in the financial market, we estimate the lower and upper bounds of turnover rate of each stock from the historical data and denote it by $\tau_{j}^{L}$ and $\tau_{j}^{U}$, respectively. That is, $\mathrm{E}\left(l_{j}\right) \in\left[\tau_{j}^{L}, \tau_{j}^{U}\right] \triangleq \bar{\tau}_{j}, \forall j$. Total turnover rate of the portfolio with turnover rate as interval parameters is

$$
\sum_{j=1}^{n}\left[\tau_{j}^{L}, \tau_{j}^{U}\right] x_{j} \triangleq \sum_{j=1}^{n} \bar{\tau}_{j} x_{j}
$$

To formulate the risk function, consider the expected absolute deviation of the random rate of return of $j$ th security from its mean, i.e., $\mathrm{E}\left(\left|\mathrm{R}_{j}-\mu_{j}\right|\right)$. Since $\mu_{j} \in\left[\mu_{j}^{L}, \mu_{j}^{U}\right]$, the expected absolute deviation of the random rate of return of $j$ th security form its mean is the set

$$
\begin{aligned}
& \left\{\mathrm{E}\left(\left|\mathrm{R}_{j}-\mu_{j}\right|\right) \mid, \mu_{j} \in \bar{\mu}_{j}\right\} \\
& =\left[\min _{\mu_{j} \in\left[\mu_{j}^{L}, \mu_{j}^{U}\right]} \mathrm{E}\left(\left|\mathrm{R}_{j}-\mu_{j}\right|\right), \max _{\mu_{j} \in\left[\mu_{j}^{L}, \mu_{j}^{U}\right]} \mathrm{E}\left(\left|\mathrm{R}_{j}-\mu_{j}\right|\right)\right] \triangleq\left[q_{j}^{L}, q_{j}^{U}\right] .
\end{aligned}
$$

Hence the risk function given in Definition 3.1 is extended to an interval valued function as

$$
\max _{1 \leq j \leq n}\left\{\left[q_{j}^{L}, q_{j}^{U}\right] x_{j}\right\} .
$$

Assume that an investor wants to accept such a portfolio whose total expected turnover rate must accept the tolerance level $\left[\tau_{f i x}^{L}, \tau_{\text {fix }}^{U}\right]$, where $\tau_{\text {fix }}^{L}$ represents a fixed expected turnover rate of the portfolio that is chosen in a pessimistic frame of mind of the investor, and $\tau_{f i x}^{U}$ represents a fixed expected turnover rate of portfolio that is chosen in an optimistic frame of mind of an investor. Mathematically,

$$
\sum_{j=1}^{n}\left[\tau_{j}^{L}, \tau_{j}^{U}\right] x_{j} \succeq\left[\tau_{f i x}^{L}, \tau_{f i x}^{U}\right]
$$

Here, we propose a portfolio optimization model, whose objectives are to minimize the maximum possible expected absolute deviation of return from expected return of the security, and to maximize the expected return of the portfolio simultaneously for a given acceptable tolerance level of expected turnover rate of the portfolio. Hence the portfolio optimization model can be expressed in the following form, which we call as minimax interval portfolio model and refer to it as MMP.

$$
\begin{gathered}
\text { (MMP) } \min \left\{\max _{1 \leq j \leq n}\left\{\left[q_{j}^{L}, q_{j}^{U}\right] x_{j}\right\},-\sum_{j=1}^{n}\left[\mu_{j}^{L}, \mu_{j}^{U}\right] x_{j}\right\} \\
\text { subject to } \sum_{j=1}^{n}\left[\tau_{j}^{L}, \tau_{j}^{U}\right] x_{j} \succeq\left[\tau_{\text {fix }}^{L}, \tau_{\text {fix }}^{U}\right], \\
\sum_{j=1}^{n} x_{j}=1,0 \leq x_{j} \leq 1, \quad \forall j .
\end{gathered}
$$

This problem cannot be solved using general optimization techniques due to the presence of interval parameters. In the following section, we discuss a concept that can be considered as a fusion between random variable and a closed interval. As a result, an interval constraint becomes a chance constraint. This new concept is used in the next section to convert MMP to a deterministic equivalent, which is free from interval uncertainties.

\subsection{Understanding intervals and interval inequalities in probabilistic sense}

Some parts of the discussion of this section are inherited from authors' previous paper [39]. Let $A$ be a random variable defined over the interval $\overline{\mathbf{A}}=\left[a^{L}, a^{U}\right]$. If the decision maker gives more importance to the selection of points from $\left[a^{L}, a^{U}\right]$ towards its mid point $\frac{a^{L}+a^{U}}{2}$ then the probability of the selection of points gradually increases while moving from the boundaries $a^{L}$ and $a^{U}$ towards the midpoint of $\left[a^{L}, a^{U}\right]$ with the highest probability at mid point. In order to assume the random variable $A$, to cover the whole interval $\overline{\mathbf{A}}$, we may accept the $3 \sigma$-rule. Denote, mean of the random variable $A$ as $m_{A}=\frac{a^{L}+a^{U}}{2}=m(\overline{\mathbf{A}})$ and $3 \sigma=\frac{a^{U}-a^{L}}{2}=w(\overline{\mathbf{A}})$, i.e., $\quad \overline{\mathbf{A}}=\left[a^{L}, a^{U}\right]=\left[m_{A}-3 \sigma, m_{A}+\right.$ $3 \sigma]$. Then $\mathrm{P}\left(m_{A}-3 \sigma \leq A \leq m_{A}+3 \sigma\right)=0.9973$, while the probability of points lying outside this interval is equal to 0.0027 , i.e., $\mathrm{P}\left(A \notin\left[m_{A}-3 \sigma, m_{A}+3 \sigma\right]\right)=0.0027$. This means it is almost impossible for the random variable $A$ to accept values from outside the interval $\overline{\mathbf{A}}$. Therefore, it is 
reasonable to consider $A$ to be normally distributed random variable with mean $\frac{a^{L}+a^{U}}{2}$ and variance $\left(\frac{a^{U}-a^{L}}{6}\right)^{2}$.

This concept associates an interval with a random variable. Henceforth, we use bold capital letter with bar for interval and denote the corresponding random variable with capital letter only. For the interval $\overline{\mathbf{A}}$, we denote $A \sim \mathcal{N}\left(m(\overline{\mathbf{A}}),(w(\overline{\mathbf{A}}) / 3)^{2}\right)$, as normally distributed random variable with mean $m(\overline{\mathbf{A}})$ and variance $(w(\overline{\mathbf{A}}) / 3)^{2}$.

Closeness between two intervals can be interpreted using the afore-mentioned concept. Consider two intervals $\overline{\mathbf{A}}=$ $\left[a^{L}, a^{U}\right]$ and $\overline{\mathbf{B}}=\left[b^{L}, b^{U}\right] . \overline{\mathbf{A}}$ and $\overline{\mathbf{B}}$ are associated with the random variables $\quad A \sim \mathcal{N}\left(m(\overline{\mathbf{A}}),(w(\overline{\mathbf{A}}) / 3)^{2}\right) \quad$ and $B \sim \mathcal{N}\left(m(\overline{\mathbf{B}}),(w(\overline{\mathbf{B}}) / 3)^{2}\right)$, respectively. Since the set of intervals is not totally ordered, closeness of $\overline{\mathbf{A}}$ and $\overline{\mathbf{B}}$ can be represented using a partial ordering. $\overline{\mathbf{A}} \preceq \overline{\mathbf{B}}$ means $a \leq b, \forall a \in \overline{\mathbf{A}}, b \in \overline{\mathbf{B}}$. Either $\overline{\mathbf{A}}$ may overlap with $\overline{\mathbf{B}}$ or $\overline{\mathbf{A}} \subseteq$ $\overline{\mathbf{B}}$ or $\overline{\mathbf{A}} \cap \overline{\mathbf{B}}=\phi$. The partial ordering $\overline{\mathbf{A}} \preceq \overline{\mathbf{B}}$ indicates certain degree of closeness of $\overline{\mathbf{A}}$ with $\overline{\mathbf{B}}$. Denote $\overline{\mathbf{C}}=\overline{\mathbf{A}} \ominus_{M} \overline{\mathbf{B}}$. Let interval $\overline{\mathbf{C}}$ be associated with random variable $C \sim \mathcal{N}\left(m(\overline{\mathbf{C}}),(w(\overline{\mathbf{C}}) / 3)^{2}\right)$. Using (2.1) one can find $m(\overline{\mathbf{C}})=m(\overline{\mathbf{A}})-m(\overline{\mathbf{B}})$ and $w(\overline{\mathbf{C}})=|w(\overline{\mathbf{A}})-w(\overline{\mathbf{B}})|$. $\overline{\mathbf{A}} \preceq \overline{\mathbf{B}}$ is equivalent to $\overline{\mathbf{A}} \ominus_{M} \overline{\mathbf{B}} \preceq 0$. We define the degree of closeness of $\overline{\mathbf{A}}$ with $\overline{\mathbf{B}}$ as $P(C \leq 0)$. For example $\overline{\mathbf{A}}=$ $[1,3], \overline{\mathbf{B}}=[2,5]$, then $\overline{\mathbf{C}}=[1,3] \ominus_{M}[2,5]=[-2,-1]$ and it is associated with the random variable $C \sim \mathcal{N}\left(-\frac{3}{2},\left(\frac{1}{6}\right)^{2}\right)$. Hence degree of closeness of $\overline{\mathbf{A}}$ with $\overline{\mathbf{B}}$ is $P(C \leq 0)=$ $P\left(\frac{C+3 / 2}{1 / 6} \leq \frac{3 / 2}{1 / 6}\right)=\Phi\left(\frac{3 / 2}{1 / 6}\right)=0.99997$, where $\left(\frac{C+3 / 2}{1 / 6}\right)$ is a standard normal variate (SNV) with mean zero and variance 1 , and $\Phi$ is its cumulative distribution function. We say, $[1,3] \preceq[2,5]$ with degree of closeness 0.99997 .

One may observe here that comparison between two intervals is associated with a closeness factor, which involves not only upper and lower bounds but also their width in the computation. Indirectly, all the points of the intervals are involved in this process. This is the basic difference between existing methodology used to handel interval uncertainty in a portfolio optimization model with bounded parameters and the methodology of this paper.

This concept can be extended to represent an interval inequality in terms of an algebraic inequality as follows. Consider the interval inequality $\overline{\mathbf{A}} x_{1} \oplus \overline{\mathbf{B}} x_{2} \preceq \overline{\mathbf{D}}, x_{1}, x_{2} \geq 0$, $\overline{\mathbf{A}}=[1,3], \overline{\mathbf{B}}=[2,4]$ and $\overline{\mathbf{D}}=[1,5] .[1,3] x_{1} \oplus[2,4] x_{2}$ is a variable interval. Denote $\overline{\mathbf{Z}}\left(x_{1}, x_{2}\right)=\left(\overline{\mathbf{A}} x_{1} \oplus \overline{\mathbf{B}} x_{2}\right) \ominus_{M} \overline{\mathbf{D}}$ $=\left[x_{1}+2 x_{2}, 3 x_{1}+4 x_{2}\right] \ominus_{M}[1,5]$. Using (2.1), $m\left(\overline{\mathbf{Z}}\left(x_{1}, x_{2}\right)\right)$ $=2 x_{1}+3 x_{2}-3$ and $w\left(\overline{\mathbf{Z}}\left(x_{1}, x_{2}\right)\right)=x_{1}+x_{2}-2 . \overline{\mathbf{Z}}\left(x_{1}, x_{2}\right)$ can be represented as a random variable $Z\left(x_{1}, x_{2}\right) \sim \mathcal{N}$ $\left(m\left(\overline{\mathbf{Z}}\left(x_{1}, x_{2}\right)\right),\left(\frac{w\left(\overline{\mathbf{Z}}\left(x_{1}, x_{2}\right)\right)}{3}\right)^{2}\right)$. Degree of closeness of the variable interval $\overline{\mathbf{A}} x_{1} \oplus \overline{\mathbf{B}} x_{2}$ with $\overline{\mathbf{D}}$ is $\mathrm{P}\left(Z\left(x_{1}, x_{2}\right) \leq 0\right)$, whose value is different for different values of $x_{1}$ and $x_{2}$. Hence, we assign a bound $\lambda \in[0,1]$ to the closeness degree of $\overline{\mathbf{A}} x_{1} \oplus \overline{\mathbf{B}} x_{2}$ with $\overline{\mathbf{D}}$, and consider the values of $\left(x_{1}, x_{2}\right)$ satisfying the interval inequality $\overline{\mathbf{A}} x_{1} \oplus \overline{\mathbf{B}} x_{2} \preceq \overline{\mathbf{D}}$ with at least degree of closeness of $\lambda$, and call such a solution as a $\lambda$-acceptable solution. For a given value of $\lambda$, the set of $\lambda$ acceptable solutions of the interval inequality $\overline{\mathbf{A}} x_{1} \oplus \overline{\mathbf{B}} x_{2} \preceq$ $\overline{\mathbf{D}}$ can be mathematically expressed as

$$
\begin{aligned}
& \left\{\left(x_{1}, x_{2}\right) \in \mathfrak{R}_{+}^{2} \mid \overline{\mathbf{A}} x_{1} \oplus \overline{\mathbf{B}} x_{2} \preceq \overline{\mathbf{D}}\right\} \\
& \equiv\left\{\left(x_{1}, x_{2}\right) \in \mathfrak{R}_{+}^{2} \mid \mathrm{P}\left(Z\left(x_{1}, x_{2}\right) \leq 0\right) \geq \lambda\right\}
\end{aligned}
$$

For example if $\lambda=0.3$, then $P\left(Z\left(x_{1}, x_{2}\right) \leq 0\right) \geq 0.3$ is equivalent to

$$
\begin{aligned}
& P\left(\frac{Z\left(x_{1}, x_{2}\right)-m\left(\overline{\mathbf{Z}}\left(x_{1}, x_{2}\right)\right)}{\frac{w\left(\overline{\mathbf{Z}}\left(x_{1}, x_{2}\right)\right)}{3}} \leq \frac{-m\left(\overline{\mathbf{Z}}\left(x_{1}, x_{2}\right)\right)}{\frac{w\left(\overline{\mathbf{Z}}\left(x_{1}, x_{2}\right)\right)}{3}}\right) \geq 0.3 \\
& \equiv \Phi\left(\frac{-m\left(\overline{\mathbf{Z}}\left(x_{1}, x_{2}\right)\right)}{\frac{w\left(\overline{\mathbf{Z}}\left(x_{1}, x_{2}\right)\right)}{3}}\right) \geq 0.3 \\
& \equiv \frac{-6 x_{1}-9 x_{2}+9}{x_{1}+x_{2}-2} \geq \Phi^{-1}(0.3) \\
& \equiv 7.04 x_{1}+10.04 x_{2} \leq 11.08 .
\end{aligned}
$$

Hence the interval inequality $[1,3] x_{1} \oplus[2,4] x_{2} \preceq[1,5]$ can be expressed as an algebraic inequality $7.04 x_{1}+$ $10.04 x_{2} \leq 11.08$ with degree of closeness $\lambda=0.3$ and solution set as $\left\{\left(x_{1}, x_{2}\right) \mid 7.04 x_{1}+10.04 x_{2} \leq 11.08, x_{1} \geq\right.$ $\left.0, x_{2} \geq 0\right\}$.

\subsection{MMP in deterministic form}

Following the discussion of section 3.2, MMP can be transformed to a general nonlinear programming as follows. The initial interval portfolio optimization model MMP, (3.23.4), is to find $y^{L}$ and $y^{U}$ in $\mathfrak{R}$, with $y^{L} \leq y^{U}$, so as to find

$$
\begin{aligned}
& \min \left\{\left[y^{L}, y^{U}\right],-\sum_{j=1}^{n}\left[\mu_{j}^{L}, \mu_{j}^{U}\right] x_{j}\right\} \\
& \text { subject to }\left[q_{j}^{L}, q_{j}^{U}\right] x_{j} \preceq\left[y^{L}, y^{U}\right], \quad j=1,2, \ldots, n \\
& \sum_{j=1}^{n}\left[\tau_{j}^{L}, \tau_{j}^{U}\right] x_{j} \succeq\left[\tau_{\text {fix }}^{L}, \tau_{\text {fix }}^{U}\right] \\
& \sum_{j=1}^{n} x_{j}=1,0 \leq x_{j} \leq 1, \forall j .
\end{aligned}
$$

This is a bi-objective linear programming problem with interval parameters, which can be converted to a single objective model using a weight $0 \leq \omega \leq 1$ as follows: 


$$
\begin{gathered}
\left(\mathbf{M M P}_{\omega}\right) \min \omega\left[y^{L}, y^{U}\right] \oplus(1-\omega)\left(-\sum_{j=1}^{n}\left[\mu_{j}^{L}, \mu_{j}^{U}\right] x_{j}\right) \\
\text { subject to }\left[q_{j}^{L}, q_{j}^{U}\right] x_{j} \preceq\left[y^{L}, y^{U}\right], \quad j=1,2, \ldots, n, \\
\sum_{j=1}^{n}\left[\tau_{j}^{L}, \tau_{j}^{U}\right] x_{j} \succeq\left[\tau_{f i x}^{L}, \tau_{f i x}^{U}\right] \\
\sum_{j=1}^{n} x_{j}=1,0 \leq x_{j} \leq 1, \forall j .
\end{gathered}
$$

Remark 3.1 Here, $\omega$ can be interpreted as the risk aversion factor of the investor. Greater the factor $\omega$, the more risk aversion the investor has. If $\omega=1$ then the investor would consider only the risk of his/her investment without paying attention to the return of the investment. Conversely, $\omega=0$ means that the investor is perusing the return of the investment only.

As per the earlier discussion in section 3.2, the interval inequalities (3.6) and (3.7) can be transformed to algebraic inequalities as follows.

Denote $\overline{\mathbf{Q}}_{j}=\left[q_{j}^{L}, q_{j}^{U}\right], \quad \overline{\mathbf{Y}}=\left[y^{L}, y^{U}\right], \bar{\tau}_{j}=\left[\tau_{j}^{L}, \tau_{j}^{U}\right], j=$ $1,2, \ldots, n$ and $\bar{\tau}_{f i x}=\left[\tau_{f i x}^{L}, \tau_{f i x}^{U}\right]$. The intervals $\overline{\mathbf{Q}}_{j}, \overline{\mathbf{Y}}, \bar{\tau}_{j}$ and $\bar{\tau}_{f i x}$ are associated with independent random variables

$$
\begin{aligned}
Q_{j} & \sim \mathcal{N}\left(m(\overline{\mathbf{Q}}),\left(\frac{w(\overline{\mathbf{Q}})}{3}\right)^{2}\right), \quad Y \sim \mathcal{N}\left(m(\overline{\mathbf{Y}}),\left(\frac{w(\overline{\mathbf{Y}})}{3}\right)^{2}\right), \\
\tau_{j} & \sim \mathcal{N}\left(m\left(\bar{\tau}_{j}\right),\left(\frac{w\left(\bar{\tau}_{j}\right)}{3}\right)^{2}\right) \text { and } \tau_{f i x} \\
& \sim \mathcal{N}\left(m\left(\bar{\tau}_{f i x}\right),\left(\frac{w\left(\bar{\tau}_{f i x}\right)}{3}\right)^{2}\right),
\end{aligned}
$$

$j=1,2, \ldots, n$, respectively. (Here we consider independent stocks to simplify the calculations.) Suppose that the variable interval $\overline{\mathbf{Q}}_{j} x_{j} \ominus_{M} \overline{\mathbf{Y}}$ is associated with the random variable $G_{j}\left(x_{j}, y^{L}, y^{U}\right)$, where $G_{j}\left(x_{j}, y^{L}, y^{U}\right) \sim \mathcal{N}\left(\mathrm{E}\left[G_{j}\left(x_{j}\right.\right.\right.$, $\left.\left.\left.y^{L}, y^{U}\right)\right], \operatorname{Var}\left[G_{j}\left(x_{j}, y^{L}, y^{U}\right)\right]\right)$. Then, for $j=1,2, \ldots, n$,

$$
\left.\begin{array}{c}
\mathrm{E}\left[G_{j}\left(x_{j}, y^{L}, y^{U}\right)\right]=m\left(\overline{\mathbf{Q}}_{j}\right) x_{j}-m(\overline{\mathbf{Y}}), \\
\operatorname{Var}\left[G_{j}\left(x_{j}, y^{L}, y^{U}\right)\right]=\left(\frac{w\left(\overline{\mathbf{Q}}_{j}\right)}{3}\right)^{2} x_{j}^{2}+\left(\frac{w(\overline{\mathbf{Y}})}{3}\right)^{2} \cdot
\end{array}\right\}
$$

Suppose the interval $\sum_{j=1}^{n} \bar{\tau}_{j} x_{j} \ominus_{M} \bar{\tau}_{f i x}$ is associated with a random variable $H\left(x_{1}, x_{2}, \ldots, x_{n}\right)$, where $H\left(x_{1}, x_{2}, \ldots\right.$, $\left.x_{n}\right) \sim \mathcal{N}\left(\mathrm{E}\left[H\left(x_{1}, x_{2}, \ldots, x_{n}\right)\right], \operatorname{Var}\left[H\left(x_{1}, x_{2}, \ldots, x_{n}\right)\right]\right)$. Then

$$
\mathrm{E}\left[H\left(x_{1}, x_{2}, \ldots, x_{n}\right)\right]=\sum_{j=1}^{n} m\left(\bar{\tau}_{j}\right) x_{j}-m\left(\bar{\tau}_{f i x}\right),
$$

$$
\operatorname{Var}\left[H\left(x_{1}, x_{2}, \ldots, x_{n}\right)\right]=\sum_{j=1}^{n}\left(\frac{w\left(\bar{\tau}_{j}\right)}{3}\right)^{2} x_{j}^{2}+\left(\frac{w\left(\bar{\tau}_{f i x}\right)}{3}\right)^{2}
$$

The feasible region of $\mathbf{M M P} \mathbf{P}_{\omega}$ is

$$
\begin{array}{r}
\Omega^{\omega}=\left\{\left(x_{1}, x_{2}, \ldots, x_{n}\right) \mid \overline{\mathbf{Q}}_{j} x_{j} \preceq \overline{\mathbf{Y}}, \sum_{j=1}^{n} \bar{\tau}_{j} x_{j} \succeq \bar{\tau}_{f i x},\right. \\
\left.\sum_{j=1}^{n} x_{j}=1, x_{j} \geq 0, j=1,2, \ldots, n\right\} .
\end{array}
$$

If we assign a bound $\lambda \in[0,1]$ for the degree of closeness of the interval inequalities in (3.6) and (3.7), then

$$
\begin{aligned}
\left\{x_{j} \mid \overline{\mathbf{Q}}_{j} x_{j} \preceq \overline{\mathbf{Y}}\right. & \text { is } \text { satisfied with the least } \\
& \text { degree of closeness } \lambda\} . \\
= & \left\{x_{j} \mid \mathrm{P}\left(G_{j}\left(x_{j}, y^{L}, y^{U}\right) \leq 0\right) \geq \lambda\right\} .
\end{aligned}
$$

Similarly,

$$
\left\{\left(x_{1}, x_{2}, \ldots, x_{n}\right) \mid \sum_{j=1}^{n} \bar{\tau}_{j} x_{j} \succeq \bar{\tau}_{f i x}\right.
$$

is satisfied with the least degree of closeness $\lambda\}$.

$$
=\left\{\left(x_{1}, x_{2}, \ldots, x_{n}\right) \mid \mathrm{P}\left(H\left(x_{1}, x_{2}, \ldots, x_{n}\right) \geq 0\right) \geq \lambda\right\} .
$$

Hence, the feasible region of $\mathbf{M M P}_{\omega}$ in (3.11), with $\lambda$ acceptability can be expressed as

$$
\begin{aligned}
\Omega_{\lambda}^{\omega}=\left\{\left(x_{1}, x_{2}, \ldots x_{n}\right) \mid\right. & \mathrm{P}\left(G_{j}\left(x_{j}, y^{L}, y^{U}\right) \leq 0\right) \geq \lambda, \\
& \mathrm{P}\left(H\left(x_{1}, x_{2}, \ldots, x_{n}\right) \geq 0\right) \geq \lambda, \\
& \sum_{j=1}^{n} x_{j}=1, \\
& \left.x_{j} \geq 0, \quad j=1,2, \ldots, n .\right\} .
\end{aligned}
$$

The objective function of $\mathbf{M M P}_{\omega}$ is in the form of interval $\omega \overline{\mathbf{Y}} \oplus(1-\omega)\left(-\sum_{j=1}^{n} \overline{\boldsymbol{\mu}}_{j} x_{j}\right)$, which may be denoted by $\overline{\mathbf{O}}\left(x_{1}, x_{2}, \ldots, x_{n}, y^{L}, y^{U}\right)$ and can be associated with a random variable 


$$
\begin{aligned}
& O\left(x_{1}, x_{2}, \ldots, x_{n}, y^{L}, y^{U}\right) \\
& \sim \mathcal{N}\left(\mathrm{E}\left[O\left(x_{1}, x_{2}, \ldots, x_{n}, y^{L}, y^{U}\right)\right]\right. \\
& \left.\operatorname{Var}\left[O\left(x_{1}, x_{2}, \ldots, x_{n}, y^{L}, y^{U}\right)\right]\right)
\end{aligned}
$$

where

$$
\left.\begin{array}{c}
\mathrm{E}\left[O\left(x_{1}, x_{2}, \ldots, x_{n}, y^{L}, y^{U}\right)\right] \\
=\omega m(\overline{\mathbf{Y}})+(1-\omega)\left(-\sum_{j=1}^{n} m\left(\overline{\boldsymbol{\mu}}_{j}\right) x_{j}\right), \\
\operatorname{Var}\left[O\left(x_{1}, x_{2}, \ldots, x_{n}, y^{L}, y^{U}\right)\right] \\
=\omega^{2}\left(\frac{w(\overline{\mathbf{Y}})}{3}\right)^{2}+(1-\omega)^{2}\left(\sum_{j=1}^{n}\left(\frac{w\left(\overline{\boldsymbol{\mu}}_{j}\right)}{3}\right)^{2} x_{j}^{2}\right) .
\end{array}\right\}
$$

Using the transformation in (3.12) and (3.13), the interval portfolio selection model $\mathbf{M M P}_{\omega}$ can be transformed to a chance constraint programming problem given as

$$
\begin{aligned}
& \left(\mathbf{M M P}_{\omega}^{\prime}\right) \min O\left(x_{1}, x_{2}, \ldots, x_{n}, y^{L}, y^{U}\right) \\
& \text { subject to } \mathrm{P}\left(G_{j}\left(x_{j}, y^{L}, y^{U}\right) \leq 0\right) \geq \lambda, \\
& \\
& \mathrm{P}\left(H\left(x_{1}, x_{2}, \ldots, x_{n}\right) \geq 0\right) \geq \lambda, \\
& \sum_{j=1}^{n} x_{j}=1, x_{j} \geq 0, j=1,2, \ldots, n,
\end{aligned}
$$

with $\lambda \in[0,1]$ as pre-assigned value.

Using the chance constraint programming technique, $\mathbf{M M P}_{\omega}^{\prime}$ can be converted into a nonlinear programming problem as described below.

$$
\begin{aligned}
& \mathrm{P}\left(G_{j}\left(x_{j}, y^{L}, y^{U}\right) \leq 0\right) \geq \lambda \\
& \equiv \mathrm{P}\left(\frac{G_{j}\left(x_{j}, y^{L}, y^{U}\right)-\mathrm{E}\left[G_{j}\left(x_{j}, y^{L}, y^{U}\right)\right]}{\sqrt{\operatorname{Var}\left[G_{j}\left(x_{j}, y^{L}, y^{U}\right)\right]}}\right. \\
& \left.\leq \frac{-\mathrm{E}\left[G_{j}\left(x_{j}, y^{L}, y^{U}\right)\right]}{\sqrt{\operatorname{Var}\left[G_{j}\left(x_{j}, y^{L}, y^{U}\right)\right]}}\right) \geq \lambda, \\
& j=1,2, \ldots, n .
\end{aligned}
$$

Since $\frac{G_{j}\left(x_{j}, y^{L}, y^{U}\right)-\mathrm{E}\left[G_{j}\left(x_{j}, \nu^{L}, y^{U}\right)\right]}{\sqrt{\operatorname{Var}\left[G_{j}\left(x_{j}, y^{L}, y^{U}\right)\right]}}$ is an $\mathrm{SNV}$, for $j=1,2, \ldots, n$,

$$
\begin{aligned}
& \mathrm{P}\left(G_{j}\left(x_{j}, y^{L}, y^{U}\right) \leq 0\right) \geq \lambda \\
& \equiv \Phi\left(\frac{-\mathrm{E}\left[G_{j}\left(x_{j}, y^{L}, y^{U}\right)\right]}{\sqrt{\operatorname{Var}\left[G_{j}\left(x_{j}, y^{L}, y^{U}\right)\right]}}\right) \geq \lambda \\
& \equiv \frac{-\mathrm{E}\left[G_{j}\left(x_{j}, y^{L}, y^{U}\right)\right]}{\sqrt{\operatorname{Var}\left[G_{j}\left(x_{j}, y^{L}, y^{U}\right)\right]}} \geq \Phi^{-1}(\lambda) \\
& \equiv \mathrm{E}\left[G_{j}\left(x_{j}, y^{L}, y^{U}\right)\right]+\Phi^{-1}(\lambda) \sqrt{\operatorname{Var}\left[G_{j}\left(x_{j}, y^{L}, y^{U}\right)\right]} \leq 0 .
\end{aligned}
$$

Using the values of $\mathrm{E}\left[G_{j}\left(x_{j}, y^{L}, y^{U}\right)\right]$ and $\operatorname{Var}\left[G_{j}\left(x_{j}, y^{L}, y^{U}\right)\right]$ from (3.8), the inequality (3.14) can be expressed as

$$
m\left(\overline{\mathbf{Q}}_{j}\right) x_{j}+\Phi^{-1}(\lambda) \sqrt{\left(\frac{w\left(\overline{\mathbf{Q}}_{j}\right)}{3}\right)^{2} x_{j}^{2}+\left(\frac{w(\overline{\mathbf{Y}})}{3}\right)^{2}} \leq m(\overline{\mathbf{Y}}),
$$

$j=1,2, \ldots, n$. Similarly,

$$
\begin{aligned}
& \mathrm{P}\left(H\left(x_{1}, x_{2}, \ldots, x_{n}\right) \geq 0\right) \geq \lambda \\
& \equiv \mathrm{P}\left(\frac{H\left(x_{1}, x_{2}, \ldots, x_{n}\right)-\mathrm{E}\left[H\left(x_{1}, x_{2}, \ldots, x_{n}\right)\right]}{\sqrt{\operatorname{Var}\left[H\left(x_{1}, x_{2}, \ldots, x_{n}\right)\right]}}\right. \\
& \left.\geq \frac{-\mathrm{E}\left[H\left(x_{1}, x_{2}, \ldots, x_{n}\right)\right]}{\sqrt{\operatorname{Var}\left[H\left(x_{1}, x_{2}, \ldots, x_{n}\right)\right]}}\right) \geq \lambda .
\end{aligned}
$$

Since $\frac{H\left(x_{1}, x_{2}, \ldots, x_{n}\right)-\mathrm{E}\left[H\left(x_{1}, x_{2}, \ldots, x_{n}\right)\right]}{\sqrt{\operatorname{Var}\left[H\left(x_{1}, x_{2}, \ldots, x_{n}\right)\right]}}$ is an $\mathrm{SNV}$,

$$
\begin{aligned}
& \mathrm{P}\left(H\left(x_{1}, x_{2}, \ldots, x_{n}\right) \geq 0\right) \geq \lambda \\
& \equiv \Phi\left(\frac{-\mathrm{E}\left[H\left(x_{1}, x_{2}, \ldots, x_{n}\right)\right]}{\sqrt{\operatorname{Var}\left[H\left(x_{1}, x_{2}, \ldots, x_{n}\right)\right]}}\right) \geq \lambda \\
& \equiv \frac{-\mathrm{E}\left[H\left(x_{1}, x_{2}, \ldots, x_{n}\right)\right]}{\sqrt{\operatorname{Var}\left[H\left(x_{1}, x_{2}, \ldots, x_{n}\right)\right]}} \leq \Phi^{-1}(\lambda) \\
& \equiv \mathrm{E}\left[H\left(x_{1}, x_{2}, \ldots, x_{n}\right)\right] \\
& +\Phi^{-1} \sqrt{\operatorname{Var}\left[H\left(x_{1}, x_{2}, \ldots, x_{n}\right)\right]} \geq 0 .
\end{aligned}
$$

Using the values of $\mathrm{E}\left[H\left(x_{1}, x_{2}, \ldots, x_{n}\right)\right]$ and Var $\left[H\left(x_{1}, x_{2}, \ldots, x_{n}\right)\right]$ from (3.9) and (3.10), (3.15) can be expressed as

$$
\begin{aligned}
& \sum_{j=1}^{n} m\left(\bar{\tau}_{j}\right) x_{j}+\Phi^{-1}(\lambda) \sqrt{\sum_{j=1}^{n}\left(\frac{w\left(\bar{\tau}_{j}\right)}{3}\right)^{2} x_{j}^{2}+\left(\frac{w\left(\bar{\tau}_{f i x}\right)}{3}\right)^{2}} \\
& \geq m\left(\bar{\tau}_{f i x}\right) .
\end{aligned}
$$

As in chance constraint programming technique, minimization of an objective function involving random variable is nothing but the minimization of sum of scalar multiple of its mean and standard deviation. Hence

$$
\begin{aligned}
& \min O\left(x_{1}, x_{2}, \ldots, x_{n}, y^{L}, y^{U}\right) \\
& \equiv \min \left\{\pi_{1} \mathrm{E}\left[O\left(x_{1}, x_{2}, \ldots, x_{n}, y^{L}, y^{U}\right)\right]\right. \\
& \left.+\pi_{2} \sqrt{\operatorname{Var}\left[O\left(x_{1}, x_{2}, \ldots, x_{n}, y^{L}, y^{U}\right)\right]}\right\} \\
& =\pi_{1}\left\{\omega m(\overline{\mathbf{Y}})+(1-\omega)\left(-\sum_{j=1}^{n} m\left(\overline{\boldsymbol{\mu}}_{j}\right) x_{j}\right)\right\} \\
& +\pi_{2} \sqrt{\omega^{2}\left(\frac{w(\overline{\mathbf{Y}})}{3}\right)^{2}+(1-\omega)^{2}\left(\sum_{j=1}^{n}\left(\frac{w\left(\overline{\boldsymbol{\mu}}_{j}\right)}{3}\right)^{2} x_{j}^{2}\right)},
\end{aligned}
$$

where $\pi_{1}$ and $\pi_{2}$ are nonnegative constants representing the relative importance of mean and standard deviation of objective function. These constants can be interpreted and have physical significance. For example: 
Table 1. The stock symbol and their upper and lower bounds of expected return, mean and width of 39 stocks of NSE-50.

\begin{tabular}{|c|c|c|c|c|c|c|c|c|}
\hline \multirow[b]{2}{*}{ Stock } & \multicolumn{2}{|c|}{$\begin{array}{l}\text { Interval expected rate of return } \\
\qquad\left(\overline{\boldsymbol{\mu}}_{j}\right)\end{array}$} & \multirow[b]{2}{*}{$m\left(\overline{\boldsymbol{\mu}}_{j}\right)$} & \multirow[b]{2}{*}{$\frac{w\left(\overline{\boldsymbol{\mu}}_{j}\right)}{3}$} & \multicolumn{2}{|c|}{$\begin{array}{l}\text { Interval expected of absolute } \\
\text { deviation }\left(\overline{\mathbf{Q}}_{j}\right)\end{array}$} & \multirow[b]{2}{*}{$m\left(\overline{\mathbf{Q}}_{j}\right)$} & \multirow[b]{2}{*}{$\frac{w\left(\overline{\mathbf{Q}}_{j}\right)}{3}$} \\
\hline & $\mu_{j}^{L}$ & $\mu_{j}^{U}$ & & & $q_{j}^{L}$ & $q_{j}^{U}$ & & \\
\hline $\mathrm{ACC}$ & 0.00540 & 0.01172 & 0.00856 & 0.00105 & 0.05271 & 0.05518 & 0.05395 & 0.00041 \\
\hline AMBUJACEM & 0.01026 & 0.01335 & 0.01181 & 0.00051 & 0.06630 & 0.06686 & 0.06658 & 0.00009 \\
\hline AXISBANK & -0.00604 & -0.00101 & -0.00353 & 0.00084 & 0.09019 & 0.09533 & 0.09276 & 0.00086 \\
\hline BHEL & -0.03845 & -0.02721 & -0.03283 & 0.00187 & 0.06726 & 0.07172 & 0.06949 & 0.00074 \\
\hline BPCL & -0.00268 & 0.00382 & 0.00057 & 0.00108 & 0.06294 & 0.06828 & 0.06561 & 0.00089 \\
\hline CAIRN & -0.00192 & 0.00075 & -0.00059 & 0.00044 & 0.03871 & 0.04940 & 0.04406 & 0.00178 \\
\hline CIPLA & 0.00822 & 0.01121 & 0.00971 & 0.00050 & 0.05885 & 0.06157 & 0.06021 & 0.00045 \\
\hline COALINDIA & -0.00496 & 0.00075 & -0.00211 & 0.00095 & 0.03687 & 0.04634 & 0.04161 & 0.00158 \\
\hline DLF & -0.01980 & -0.01101 & -0.01541 & 0.00146 & 0.07887 & 0.11051 & 0.09469 & 0.00527 \\
\hline DRREDDY & 0.01367 & 0.01626 & 0.01497 & 0.00043 & 0.04250 & 0.04818 & 0.04534 & 0.00095 \\
\hline GAIL & 0.01192 & 0.01426 & 0.01309 & 0.00039 & 0.04544 & 0.05229 & 0.04886 & 0.00114 \\
\hline GRASIM & 0.00513 & 0.01192 & 0.00852 & 0.00113 & 0.05215 & 0.05403 & 0.05309 & 0.00031 \\
\hline HCLTECH & 0.02772 & 0.06599 & 0.04686 & 0.00638 & 0.05385 & 0.27081 & 0.16233 & 0.03616 \\
\hline HDFC & 0.00462 & 0.01000 & 0.00731 & 0.00090 & 0.05103 & 0.05763 & 0.05433 & 0.00110 \\
\hline HDFCBANK & 0.00992 & 0.01247 & 0.01119 & 0.00042 & 0.05648 & 0.05918 & 0.05783 & 0.00045 \\
\hline HINDALCO & -0.01412 & -0.00740 & -0.01076 & 0.00112 & 0.06891 & 0.08832 & 0.07862 & 0.00324 \\
\hline ICICIBANK & -0.00082 & 0.00521 & 0.00219 & 0.00100 & 0.07429 & 0.07726 & 0.07577 & 0.00049 \\
\hline IDFC & -0.01439 & -0.00818 & -0.01128 & 0.00103 & 0.08318 & 0.08878 & 0.08598 & 0.00093 \\
\hline INFY & 0.00478 & 0.00826 & 0.00652 & 0.00058 & 0.05724 & 0.07827 & 0.06776 & 0.00351 \\
\hline ITC & 0.01990 & 0.02365 & 0.02177 & 0.00062 & 0.04327 & 0.05003 & 0.04665 & 0.00113 \\
\hline KOTAKBANK & 0.01386 & 0.01774 & 0.01580 & 0.00065 & 0.04925 & 0.06973 & 0.05949 & 0.00341 \\
\hline LT & -0.01051 & -0.00440 & -0.00746 & 0.00102 & 0.07349 & 0.07674 & 0.07512 & 0.00054 \\
\hline LUPIN & 0.02283 & 0.02589 & 0.02436 & 0.00051 & 0.04753 & 0.05578 & 0.05166 & 0.00138 \\
\hline M\&M & 0.02543 & 0.02865 & 0.02704 & 0.00054 & 0.04491 & 0.05060 & 0.04775 & 0.00095 \\
\hline MARUTI & 0.00474 & 0.00813 & 0.00644 & 0.00056 & 0.07128 & 0.08377 & 0.07753 & 0.00208 \\
\hline NMDC & -0.02308 & -0.01542 & -0.01925 & 0.00128 & 0.07428 & 0.07458 & 0.07443 & 0.00005 \\
\hline NTPC & -0.01032 & -0.00787 & -0.00910 & 0.00041 & 0.03736 & 0.05453 & 0.04595 & 0.00286 \\
\hline ONGC & -0.00544 & 0.00102 & -0.00221 & 0.00108 & 0.05693 & 0.05728 & 0.05710 & 0.00006 \\
\hline PNB & 0.02224 & 0.03411 & 0.02817 & 0.00198 & 0.08577 & 0.09019 & 0.08798 & 0.00074 \\
\hline POWERGRID & -0.00198 & 0.00101 & -0.00048 & 0.00050 & 0.04078 & 0.04109 & 0.04093 & 0.00005 \\
\hline RANBAXY & -0.01001 & 0.00159 & -0.00421 & 0.00193 & 0.08190 & 0.08995 & 0.08592 & 0.00134 \\
\hline RELIANCE & -0.00134 & 0.00056 & -0.00039 & 0.00032 & 0.06272 & 0.06429 & 0.06351 & 0.00026 \\
\hline RELINFRA & -0.02599 & -0.01801 & -0.02200 & 0.00133 & 0.08362 & 0.12002 & 0.10182 & 0.00607 \\
\hline SBIN & -0.02351 & -0.00608 & -0.01479 & 0.00290 & 0.07460 & 0.07686 & 0.07573 & 0.00038 \\
\hline SESAGOA & -0.02351 & -0.00729 & -0.01540 & 0.00270 & 0.07460 & 0.08369 & 0.07915 & 0.00151 \\
\hline SUNPHARMA & 0.03078 & 0.03531 & 0.03305 & 0.00076 & 0.05049 & 0.05412 & 0.05230 & 0.00060 \\
\hline TATAPOWER & -0.01197 & -0.00808 & -0.01003 & 0.00065 & 0.06669 & 0.07386 & 0.07027 & 0.00119 \\
\hline TATASTEEL & -0.01799 & -0.01004 & -0.01401 & 0.00133 & 0.07029 & 0.09891 & 0.08460 & 0.00477 \\
\hline TCS & 0.02350 & 0.02683 & 0.02516 & 0.00056 & 0.05453 & 0.05511 & 0.05482 & 0.00010 \\
\hline
\end{tabular}

$\pi_{1}=0$ represents that the decision maker's aim is to optimize the variation of objective function about mean without caring about mean of the objective function;

$\pi_{2}=0$ indicates that the mean value of objective function is optimized without bothering about the variation of objective function.
Similarly, $\pi_{1}=\pi_{2}>0$ means that the decision maker is giving equal importance to both. With this background the original interval portfolio selection problem MMP can be stated as a deterministic nonlinear programming problem as follows, which is free from interval uncertainty: 
Table 2. The upper and lower bounds of expected turnover rate, mean and width of interval of 39 stocks of NSE-50.

\begin{tabular}{|c|c|c|c|c|c|c|c|c|c|}
\hline \multirow[b]{2}{*}{ Stock } & \multicolumn{2}{|c|}{$\begin{array}{l}\text { Interval expected liquidity } \\
\qquad\left(\bar{\tau}_{j}\right)\end{array}$} & \multirow[b]{2}{*}{$m\left(\bar{\tau}_{j}\right)$} & \multirow[b]{2}{*}{$\frac{w\left(\bar{\tau}_{j}\right)}{3}$} & \multirow[b]{2}{*}{ Stock } & \multicolumn{2}{|c|}{$\begin{array}{l}\text { Interval expected of liquidity } \\
\qquad\left(\bar{\tau}_{j}\right)\end{array}$} & \multirow[b]{2}{*}{$m\left(\bar{\tau}_{j}\right)$} & \multirow[b]{2}{*}{$\frac{w\left(\bar{\tau}_{j}\right)}{3}$} \\
\hline & $\tau_{j}^{L}$ & $\tau_{j}^{U}$ & & & & $\tau_{j}^{L}$ & $\tau_{j}^{U}$ & & \\
\hline $\mathrm{ACC}$ & 0.08998 & 0.87501 & 0.48250 & 0.13084 & KOTAKBANK & 0.15808 & 0.95772 & 0.55790 & 0.13327 \\
\hline AMBUJACEM & 0.14131 & 0.90099 & 0.52115 & 0.12661 & LT & 0.16255 & 0.74657 & 0.45456 & 0.09734 \\
\hline AXISBANK & 0.11438 & 0.74338 & 0.42888 & 0.10483 & LUPIN & 0.15930 & 0.86490 & 0.51210 & 0.11760 \\
\hline BHEL & 0.18527 & 0.87774 & 0.53151 & 0.11541 & $\mathrm{M} \& \mathrm{M}$ & 0.18100 & 0.90981 & 0.54541 & 0.12147 \\
\hline BPCL & 0.08680 & 0.79925 & 0.44303 & 0.11874 & MARUTI & 0.07109 & 0.82286 & 0.44697 & 0.12529 \\
\hline CAIRN & 0.19560 & 0.96358 & 0.57959 & 0.12800 & NMDC & 0.12804 & 0.93938 & 0.53371 & 0.13522 \\
\hline CIPLA & 0.21452 & 0.90606 & 0.56029 & 0.11526 & NTPC & 0.32424 & 0.91785 & 0.62105 & 0.09894 \\
\hline COALINDIA & 0.20782 & 0.93612 & 0.57197 & 0.12138 & ONGC & 0.27551 & 0.85127 & 0.56339 & 0.09596 \\
\hline DLF & 0.05422 & 0.60115 & 0.32768 & 0.09115 & PNB & 0.09949 & 0.79828 & 0.44888 & 0.11646 \\
\hline DRREDDY & 0.23460 & 0.90995 & 0.57228 & 0.11256 & POWERGRID & 0.18262 & 0.93139 & 0.55701 & 0.12480 \\
\hline GAIL & 0.11770 & 0.88664 & 0.50217 & 0.12816 & RANBAXY & 0.09051 & 0.75591 & 0.42321 & 0.11090 \\
\hline GRASIM & 0.12987 & 0.92106 & 0.52547 & 0.13187 & RELIANCE & 0.18926 & 0.81909 & 0.50418 & 0.10497 \\
\hline HCLTECH & 0.17914 & 0.91745 & 0.54830 & 0.12305 & RELINFRA & 0.04792 & 0.73441 & 0.39117 & 0.11442 \\
\hline HDFC & 0.35793 & 0.87267 & 0.61530 & 0.08579 & SBIN & 0.08443 & 0.59869 & 0.34156 & 0.08571 \\
\hline HDFCBANK & 0.22471 & 0.94115 & 0.58293 & 0.11941 & SESAGOA & 0.09721 & 0.62596 & 0.36159 & 0.08813 \\
\hline HINDALCO & 0.13643 & 0.75591 & 0.44617 & 0.10325 & SUNPHARMA & 0.21595 & 0.90155 & 0.55875 & 0.11427 \\
\hline ICICIBANK & 0.16941 & 0.80131 & 0.48536 & 0.10532 & TATAPOWER & 0.15338 & 0.92826 & 0.54082 & 0.12915 \\
\hline IDFC & 0.19809 & 0.80536 & 0.50173 & 0.10121 & TATASTEEL & 0.10429 & 0.60760 & 0.35595 & 0.08388 \\
\hline INFY & 0.20061 & 0.86729 & 0.53395 & 0.11111 & TCS & 0.18220 & 0.89927 & 0.54073 & 0.11951 \\
\hline ITC & 0.28197 & 0.88795 & 0.58496 & 0.10100 & & & & & \\
\hline
\end{tabular}

$\left(\mathbf{M M P}_{\left(\omega ; \pi_{1}, \pi_{2}\right)}\right)$

$\min \left[\pi_{1}\left\{\omega m(\overline{\mathbf{Y}})+(1-\omega)\left(-\sum_{j=1}^{n} m\left(\overline{\boldsymbol{\mu}}_{j}\right) x_{j}\right)\right\}\right.$

$\left.+\pi_{2} \sqrt{\omega^{2}\left(\frac{w(\overline{\mathbf{Y}})}{3}\right)^{2}+(1-\omega)^{2}\left(\sum_{j=1}^{n}\left(\frac{w\left(\overline{\boldsymbol{\mu}}_{j}\right)}{3}\right)^{2} x_{j}^{2}\right)}\right]$,

subject to

$m\left(\overline{\mathbf{Q}}_{j}\right) x_{j}+\Phi^{-1}(\lambda) \sqrt{\left(\frac{w\left(\overline{\mathbf{Q}}_{j}\right)}{3}\right)^{2} x_{j}^{2}+\left(\frac{w(\overline{\mathbf{Y}})}{3}\right)^{2}} \leq m(\overline{\mathbf{Y}})$,

$\sum_{j=1}^{n} m\left(\bar{\tau}_{j}\right) x_{j}+\Phi^{-1}(\lambda) \sqrt{\sum_{j=1}^{n}\left(\frac{w\left(\bar{\tau}_{j}\right)}{3}\right)^{2} x_{j}^{2}+\left(\frac{w\left(\bar{\tau}_{f i x}\right)}{3}\right)^{2}} \geq m\left(\bar{\tau}_{f i x}\right)$,

$\sum_{j=1}^{n} x_{j}=1, \quad 0 \leq x_{j} \leq 1, j=1,2, \ldots, n$.

For pre-assigned values of $\omega, \pi_{1}$ and $\pi_{2}$ by the investor, suppose the solution of the model $\mathbf{M M P}_{\left(\omega ; \pi_{1}, \pi_{2}\right)}$ is $\left(\mathbf{x}^{*}, y^{L^{*}}, y^{R^{*}}\right)_{\left(\omega ; \pi_{1}, \pi_{2}\right)}$. This indicates that $\mathbf{x}^{*}$ is an efficient portfolio for MMP with maximum range of risk lying in $\left[y^{L^{*}}, y^{R^{*}}\right]$. In the next section, we will explain the whole procedure by means of a simple example.

The whole procedure of obtaining a $\lambda$-acceptable efficient portfolio can be stated in the following steps.

Step 1: Input data:

(a) Number of stocks $(n)$. (b) Historical opening $\left(p_{j t}^{\text {open }}\right)-, \quad$ maxi$\operatorname{mum}\left(p_{j t}^{\max }\right)-$, minimum $\left(p_{j t}^{\min }\right)-$ and closing $\left(p_{j t}^{\text {close }}\right)$ - prices of each stock for given time period $(T)$.

(c) Daily turnover volume and tradeable volume of each stock for complete time period (T).

(d) The minimum and maximum tolerance levels of expected return $\mu_{f i x}^{L}$ and $\mu_{f i x}^{U}$, respectively.

(e) Values of $\lambda, \omega, \pi_{1}$ and $\pi_{2}$.

Step 2: (a) Calculate rate of returns corresponding to each price $r_{j t}^{t y p e}=\frac{p_{j t}^{t y p e}-p_{j t-1}^{t y p e}}{p_{j t-1}^{t y p e}}$, for $j=1,2, \ldots, n$, $t=1,2, \ldots, T$ and type $\in\{$ open, $\max , \min$, close $\}$.

(b) Calculate $\mu_{j}^{\text {type }}=\frac{1}{T} \sum_{t=1}^{T} r_{j t}^{\text {type }}$, for $j=1$, $2, \ldots, n$.

Step 3: (a) Estimate $\mu_{j}^{L}=\min \left\{\mu_{j}^{\text {open }}, \mu_{j}^{\max }, \mu_{j}^{\text {min }}, \mu_{j}^{\text {close }}\right\}$, $\mu_{j}^{U}=\max \left\{\mu_{j}^{\text {open }}, \mu_{j}^{\max }, \mu_{j}^{\min }, \mu_{j}^{\text {close }}\right\}$, for $j=$ $1,2, \ldots, n$.

(a) Calculate $m\left(\overline{\boldsymbol{\mu}}_{j}\right)=\frac{\mu_{j}^{L}+\mu_{j}^{U}}{2}$ and $w\left(\overline{\boldsymbol{\mu}}_{j}\right)=\frac{\mu_{j}^{U}-\mu_{j}^{L}}{2}$, for $j=1,2, \ldots, n$. 


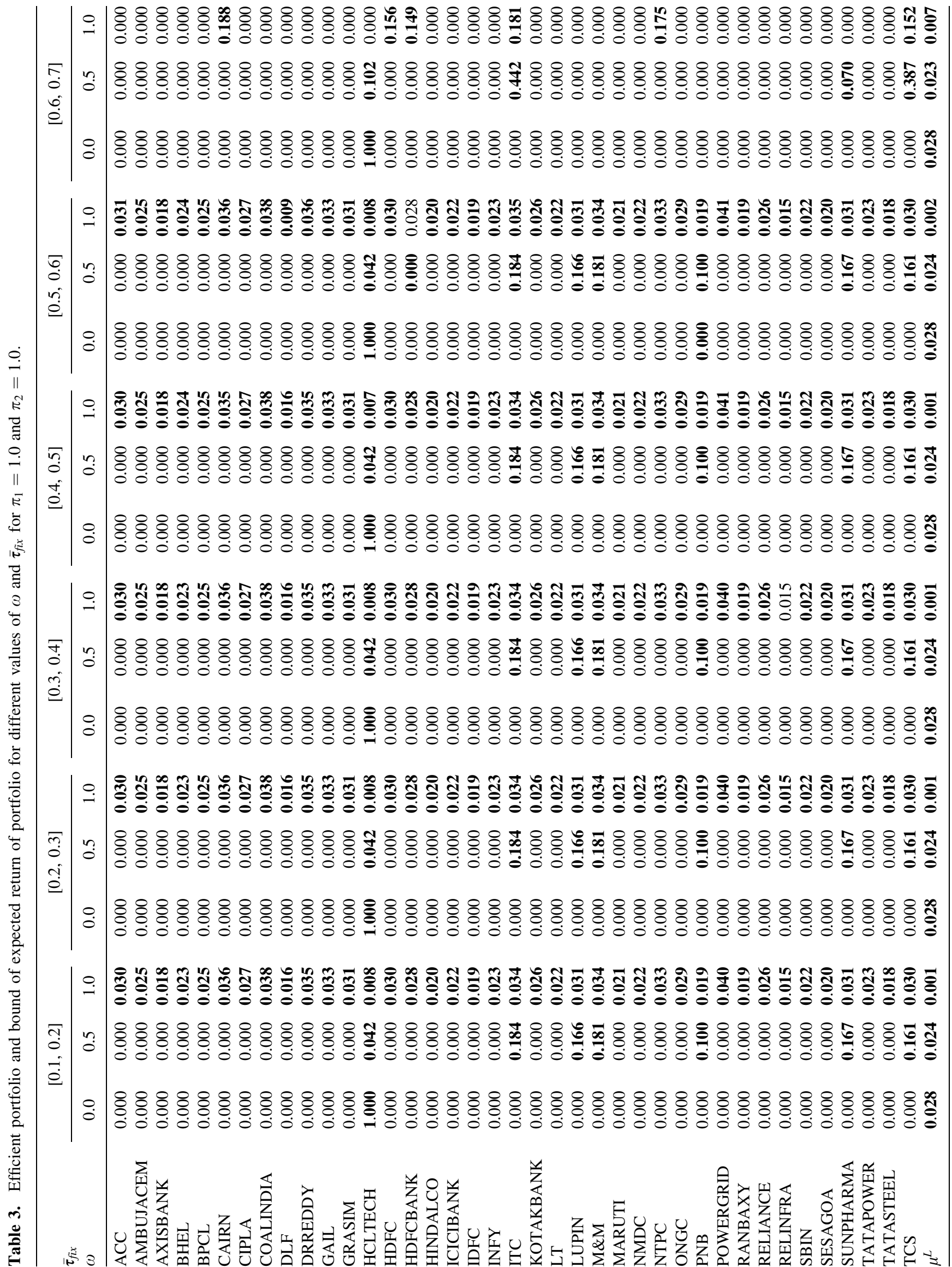


Step 4: (a) Compute

$q_{j}^{L}=\frac{1}{T} \sum_{t=1}^{T}\left|\hat{r}_{j t}^{L}-\mu_{j}^{L}\right|$ and $q_{j}^{U}=\frac{1}{T} \sum_{t=1}^{T}\left|\hat{r}_{j t}^{U}-\mu_{j}^{U}\right|$,

$j=1,2, \ldots, n$, where $\hat{r}_{j t}^{L}$ represents return of $j$ th stock at the time $t$ corresponding to the lower bound $\left(\mu_{j}^{L}\right)$ of expected return obtained. Similarly, $\hat{r}_{j t}^{U}$ represents return of $j$ th stock at the time $t$ corresponding to the upper bound $\left(\mu_{j}^{U}\right)$ of expected return obtained.

(b) If $q_{j}^{L}>q_{j}^{U}$ then set $q_{j}^{L}=q_{j}^{U}$, otherwise $q_{j}^{U}=q_{j}^{L}$.

(c) Calculate $m\left(\overline{\mathbf{Q}}_{j}\right)=\frac{q_{j}^{L}+q_{j}^{U}}{2}$ and $w\left(\overline{\mathbf{Q}}_{j}\right)=\frac{q_{j}^{U}-q_{j}^{L}}{2}$, for $j=1,2, \ldots, n$.

Step 5: (a) Calculate daily turnover rate

$$
\left(\tau_{j t}\right)=\frac{\text { turnover volume }}{\text { tradable volume }}, \forall j, t .
$$

(a) Estimate $\quad \tau_{j}^{L}=\min _{1 \leq t \leq T}\left\{\tau_{j t}\right\}, \quad$ and $\tau_{j}^{U}=\max _{1 \leq t \leq T}\left\{\tau_{j t}\right\}$, for $j=1,2, \ldots, n$.

Step 6: Choose $\pi_{1}, \pi_{2} \geq 0, \omega, \lambda \in[0,1]$ and solve $\mathbf{M M P}_{\left(\omega ; \pi_{1}, \pi_{2}\right)}$ using LINGO 11 ( or any other software package that supports the non-linear optimization problem) and obtain the optimal solution $\mathbf{x}^{*}=\left(x_{1}^{*}, x_{2}^{*}, \ldots, x_{n}^{*}\right)$, and $y^{L *}$ and $y^{U *}$.

Step 7: Obtain a $\lambda$-acceptable efficient portfolio $\mathbf{x}^{*}=$ $\left(x_{1}^{*}, x_{2}^{*}, \ldots, x_{n}^{*}\right)$.

\section{Empirical result}

In this section, the applicability of the proposed portfolio selection methodology for a large data set is demonstrated. Financial data for monthly opening-, maximum-, minimum- and closing-price of 39 stocks listed in National Stock Exchange (NSE), India, for a period of 3 years starting from September 1, 2013, to August 31, 2016, are collected. Expected returns for opening, maximum, minimum and closing prices of each stock are calculated. Using steps $2-4$, the bounds of expected return $\left(\overline{\boldsymbol{\mu}}_{j}\right)$ and expected absolute deviation of return $\left(\overline{\mathbf{Q}}_{j}\right)$, including the mean $\left(m\left(\overline{\boldsymbol{\mu}}_{j}\right), m\left(\overline{\mathbf{Q}}_{j}\right)\right)$ and width $\left(w\left(\overline{\boldsymbol{\mu}}_{j}\right), w\left(\overline{\mathbf{Q}}_{j}\right)\right)$ of intervals, of each stock are calculated and summarized in table 1. Here we use historical data of turnover rate to estimate the upper and lower bounds of expected turnover rate of each stock. 


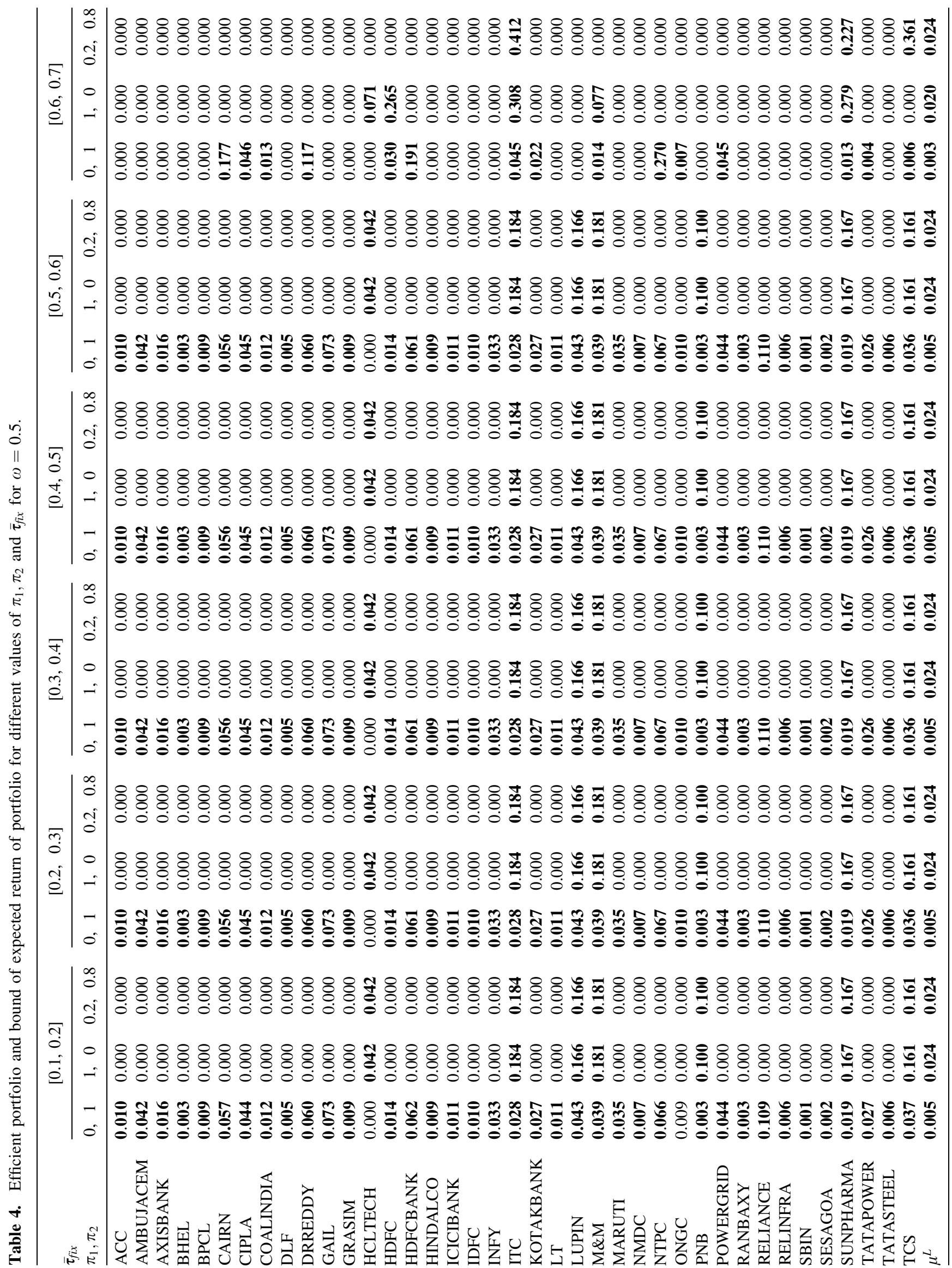


For this, daily turnover rate of each stock for the period September 1, 2013-August 31, 2016 is considered, and bounds of expected turnover rate are calculated using the step 5(b). This is summarized in table 2 .

Next we present computational results based on the afore-mentioned data for $\lambda=0.9015$ and different values of $\bar{\tau}_{f i x}, \pi_{1}, \pi_{2}$ and $\omega$. First consider $\pi_{1}=\pi_{2}=1$, and obtain $\lambda$-acceptable efficient portfolio for the different values of $\bar{\tau}_{f i x}$ and $\omega$ by solving $\mathbf{M M P} \mathbf{P}_{\left(\omega ; \pi_{1}, \pi_{2}\right)}$. The results are given in table 3.

One may observe from this table that if the objective function does not involve the risk function, i.e., $\omega=0$, then corresponding to different values of $\bar{\tau}_{f i x}=[0.1,0.2]$, $[0.2,0.3],[0.3,0.4],[0.4,0.5],[0.5,0.6]$ and $[0.6,0.7]$, we obtain a portfolio that contains only one stock with expected return in $\left[\mu^{L}, \mu^{U}\right]=[0.028,0.066]$, while the value of $\left[y^{L}, y^{U}\right]=[0,10000000]$. This indicates that high risk is involved in getting high return. If both the objectives (risk and return) get equal preferences, i.e., value of $\omega=0.5$, then portfolio contains seven stocks (which are displayed in the table in bold faces) with expected return $\left[\mu^{L}, \mu^{U}\right]=[0.024,0.030]$ and risk level $\left[y^{L}, y^{U}\right]=$ $[0.009,0.009]$ for the values of $\bar{\tau}_{f i x}=[0.1,0.2],[0.2,0.3]$, $[0.3,0.4], \quad[0.4,0.5]$ and $[0.5,0.6]$. However, for $\bar{\tau}_{f i x}=[0.6,0.7]$, portfolio consists of only four stocks with $\left[\mu^{L}, \mu^{U}\right]=[0.023,0.030]$ and $\left[y^{L}, y^{U}\right]=[0.021,0.021]$. This means that if the investor wants to select more liquid stocks with conservative mind, then expected return lies in $[0.023,0.030]$ but the risk level becomes high. However, if we assume that the investor has an aggressive and optimistic mind, i.e., $\bar{\tau}_{f i x}=[0.6,0.7]$ and $\omega=1$, then the total money is diversified into six stocks with expected return lying in $[0.007,0.010]$ with risk level 0.008 . This shows that if the investor wants to select highly liquid stocks with minimum level of risk then the expected return of portfolio will also become low.

Next, we consider the fixed value of $\omega=0.5$, and obtain $\lambda$-acceptable efficient portfolio for the different values of $\pi_{1}, \pi_{2}$ and $\bar{\tau}_{f i x}$ solving $\mathbf{M M P} \mathbf{P}_{\left(\omega ; \pi_{1}, \pi_{2}\right)}$. The results are given in table 4.

One may observe from this table that for the fixed value of $\omega=0.5$, if we change the values of $\pi_{1}, \pi_{2}$ and $\bar{\tau}_{f i x}$ then $\lambda$ acceptable efficient portfolio also changes. However, if $\pi_{1}=0$ and $\pi_{2}=1$, then $\lambda$-acceptable efficient portfolio for $\bar{\tau}_{f i x}=[0.1,0.2],[0.2,0.3],[0.3,0.4],[0.4,0.5]$ and $[0.5,0.6]$ are the same, while for $[0.6,0.7]$ it is different. This shows that if the investor wants to invest in highly liquid stocks then the return of investment will reduce but the risk level will increase. A similar observation holds for $\pi_{1}=1, \pi_{2}=$ 0 and $\pi_{1}=0.2, \pi_{2}=0.8$ corresponding to the different values of $\bar{\tau}_{f i x}$. From the earlier discussion, we find that for the given different values of $\bar{\tau}_{f i x}, \omega, \pi_{1}$ and $\pi_{2}$, one can obtain the different portfolio investment strategies by solving $\mathbf{M M P}_{\left(\omega ; \pi_{1}, \pi_{2}\right)}$ for a particular value of $\lambda \in[0,1]$. Choosing the values of the parameters $\bar{\tau}_{f i x}, \omega, \pi_{1}$ and $\pi_{2}$, 
Table 5. Efficient portfolio corresponding to Lai et al [27], Cai et al [21] and our model.

\begin{tabular}{|c|c|c|c|c|c|c|c|}
\hline Stock & Lai et al & Cai et al & Our method & Stock & Lai et al & Cai et al & Our method \\
\hline $\mathrm{ACC}$ & 0.000 & 0.000 & 0.000 & $\mathrm{LT}$ & 0.000 & 0.000 & 0.000 \\
\hline AMBUJACEM & 0.000 & 0.000 & 0.000 & LUPIN & 0.000 & 0.152 & 0.166 \\
\hline AXISBANK & 0.000 & 0.000 & 0.000 & $\mathrm{M} \& \mathrm{M}$ & 0.435 & 0.156 & 0.181 \\
\hline BHEL & 0.000 & 0.000 & 0.000 & MARUTI & 0.000 & 0.000 & 0.000 \\
\hline BPCL & 0.000 & 0.000 & 0.000 & NMDC & 0.000 & 0.000 & 0.000 \\
\hline CAIRN & 0.000 & 0.000 & 0.000 & NTPC & 0.000 & 0.000 & 0.000 \\
\hline CIPLA & 0.000 & 0.000 & 0.000 & ONGC & 0.000 & 0.000 & 0.000 \\
\hline COALINDIA & 0.000 & 0.000 & 0.000 & PNB & 0.000 & 0.323 & 0.100 \\
\hline DLF & 0.000 & 0.000 & 0.000 & POWERGRID & 0.000 & 0.000 & 0.000 \\
\hline DRREDDY & 0.000 & 0.000 & 0.000 & RANBAXY & 0.000 & 0.000 & 0.000 \\
\hline GAIL & 0.000 & 0.000 & 0.000 & RELIANCE & 0.000 & 0.000 & 0.000 \\
\hline GRASIM & 0.000 & 0.000 & 0.000 & RELIANCE & 0.000 & 0.000 & 0.000 \\
\hline HCLTECH & 0.254 & 0.075 & 0.042 & RELINFRA & 0.000 & 0.000 & 0.000 \\
\hline HDFC & 0.000 & 0.000 & 0.000 & SBIN & 0.000 & 0.000 & 0.000 \\
\hline HDFCBANK & 0.000 & 0.000 & 0.000 & SESAGOA & 0.000 & 0.000 & 0.000 \\
\hline HINDALCO & 0.000 & 0.000 & 0.000 & SUNPHARMA & 0.311 & 0.196 & 0.167 \\
\hline ICICIBANK & 0.000 & 0.000 & 0.000 & TATAPOWER & 0.000 & 0.000 & 0.000 \\
\hline IDFC & 0.000 & 0.000 & 0.000 & TATASTEEL & 0.000 & 0.000 & 0.000 \\
\hline INFY & 0.000 & 0.000 & 0.000 & TCS & 0.000 & 0.099 & 0.161 \\
\hline ITC & 0.000 & 0.000 & 0.184 & {$\left[\mu^{L}, \mu^{U}\right]$} & {$[0.027,0.030]$} & 0.033 & {$[0.024,0.030]$} \\
\hline KOTAKBANK & 0.000 & 0.000 & 0.000 & Risk level & 0.025 & 0.013 & 0.009 \\
\hline
\end{tabular}

according to the investors' frame of mind, the investor may achieve a favourite portfolio investment strategy.

\subsection{Verification}

Next, we verify our results with the result obtained using the methodologies due to Lai et al [27] and Cai et al [21]. They are provided in table 5. Since Cai et al [21] consider the deterministic form of the model, to estimate parameters of the model, we consider only the monthly closing price of all the stocks for the same time period. Corresponding to these data, we obtain an efficient portfolio using the Cai et al [21] model by assigning equal weights to objectives. For the Lai et al [27] model, we use the same data (which are used in our model) and estimate interval parameters of the model, and obtain the efficient portfolio. For our model, $\lambda$-acceptable efficient portfolio is obtained for $\lambda=0.9015$, $\omega=0.5, \pi_{1}=\pi_{2}=1$, and $\bar{\tau}_{f i x}=[0.5,0.6]$.

One may observe from table 5 that the portfolio obtained using the Lai et al [27] method contains only three stocks, viz., HCLTECH, M\&M and SUNPHARMA (see figure 1) with $\overline{\boldsymbol{\mu}}=[0.027,0.030]$ and risk level 0.025 , while the portfolio obtained using the Cai et al [21] method contains six stocks, viz., HCLTECH, LUPIN, M\&M, PNB, SUNPHARMA and TCS (see figure 2) with $\mu=0.033$ and risk level 0.013. Using our model, we get seven stocks, viz., HCLTECH, ITC, LUPIN, M\&M, PNB, SUNPHARMA and TCS (see figure 3)in the portfolio with expected return of portfolio $[0.024,0.030]$ and risk level 0.009 . This shows

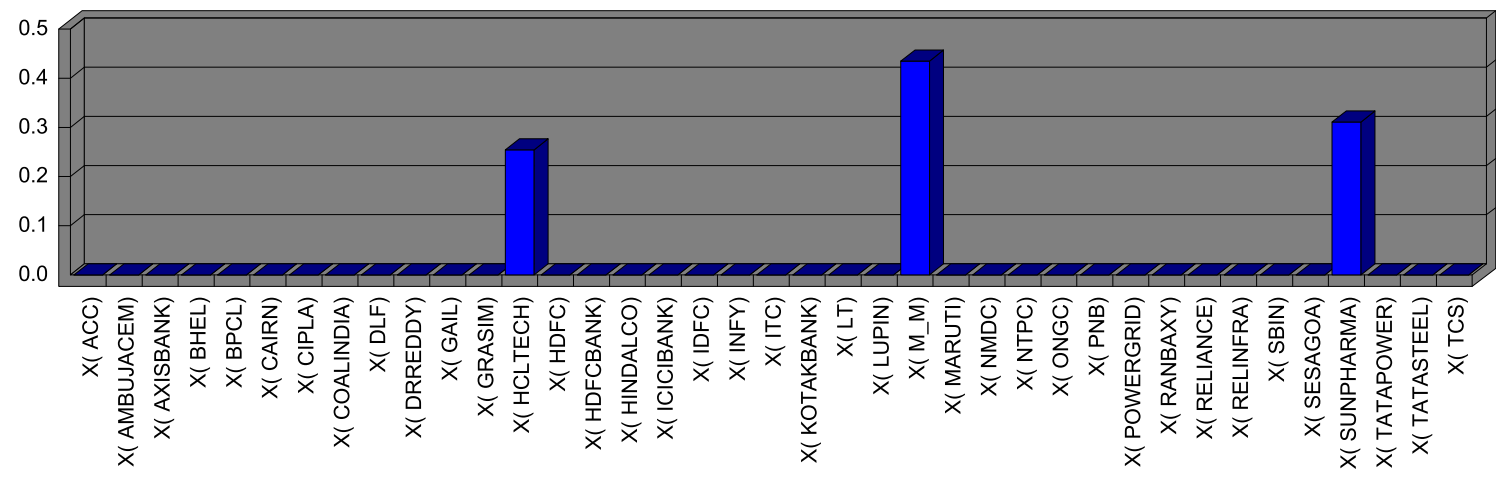

Figure 1. Portfolio obtained by Lai et al [27] method using LINGO. 

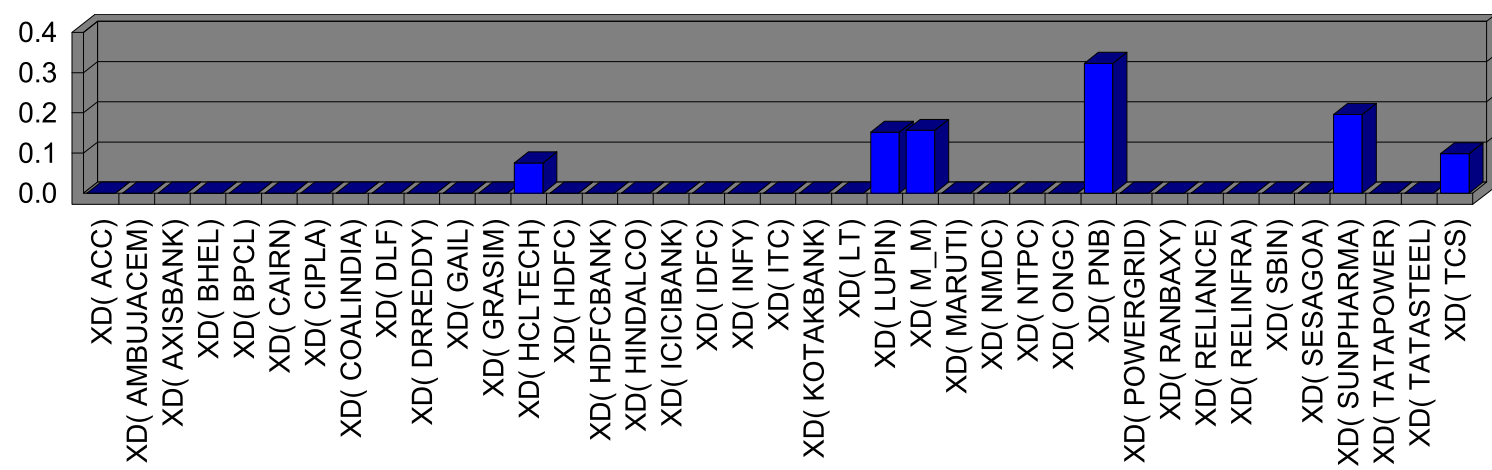

Figure 2. Portfolio obtained by Cai et al [21] method using LINGO.



Figure 3. Portfolio obtained by our method using LINGO.

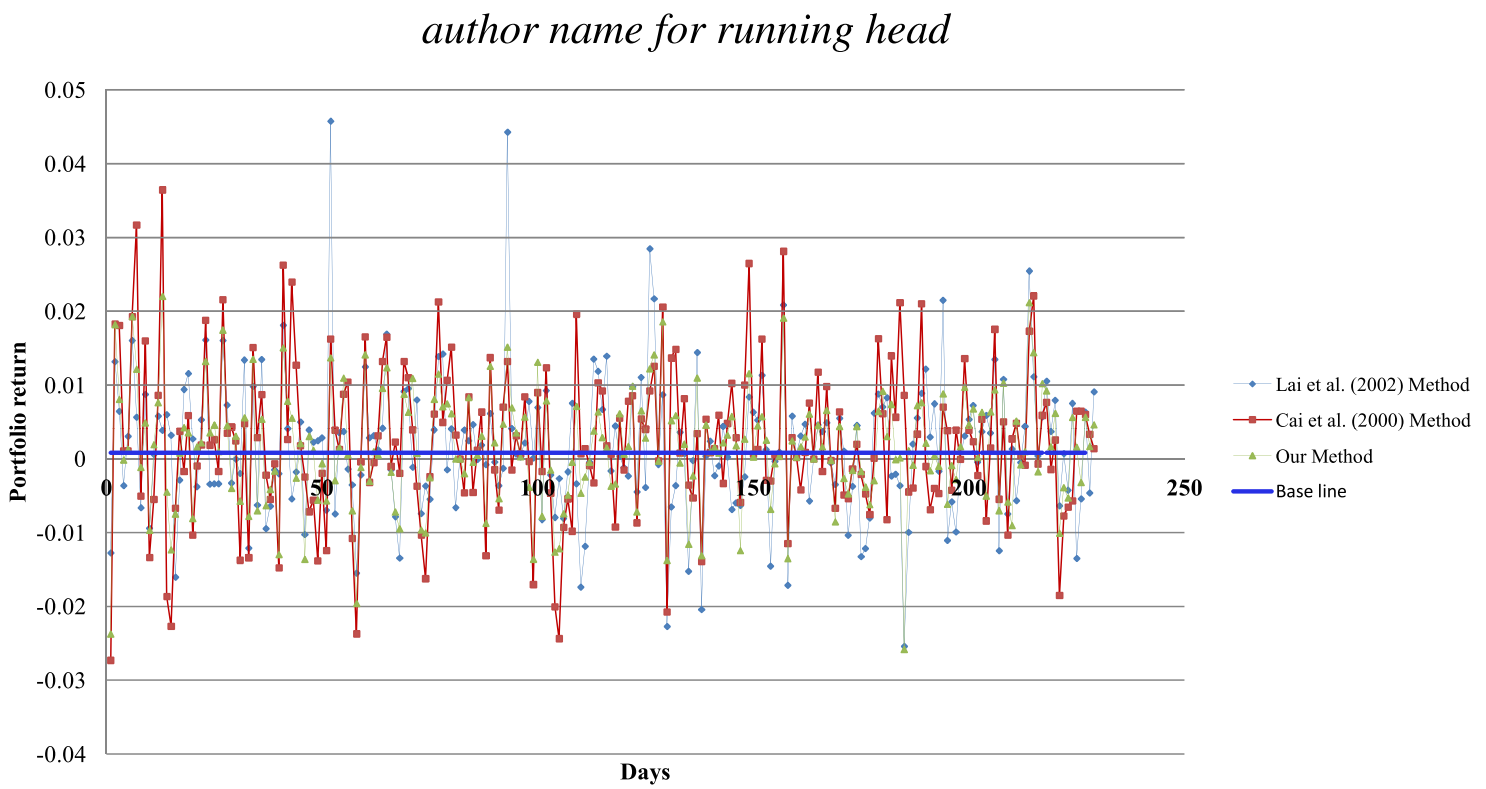

Figure 4. Actual daily portfolio returns corresponding to Lai et al [27], Cai et al [21] and our method.

that the portfolio obtained using our method has reduced the risk of portfolio as compared with both Lai et al [27] and Cai et al [21] models.
Further, in order to show the effectiveness of investment strategies obtained by Lai et al [27], Cai et al [21] and our method given in table 5, we have considered the actual 
daily return rate corresponding to adjusted closing price of selected stocks for the period September 1, 2013-July 31, 2016. We have calculated actual daily return of the portfolio using the investment strategies obtained by Lai et al [27], Cai et al [21] and our method. The graphical representation of actual daily portfolio return corresponding to each method is given in figure 4 .

One may observe from figure 4 that the fluctuations of returns obtained by Lai et al [27] and Cai et al [21] methods are more in comparison with our method from the base line of expected return. We consider lower bound of expected return of portfolio as the base line of expected return obtained by our method, i.e., 0.0008 daily basis, since it is almost equal to lower bound of expected return obtained by Lai et al [27] method and expected return obtained by Cai et al [21] method. This means that portfolios obtained by our method are more consistent than returns obtained by both Lai et al [27] and Cai et al [21] method.

\section{Conclusion}

In this paper, we have studied a portfolio selection problem with minimax rule wherein the expected return, liquidity and risk vary in closed intervals. An acceptable efficient portfolio of the model is obtained by associating a random variable with a general interval, and chance constraint with an interval constraint. The computational results show that the proposed model provides a more efficient portfolio according to the investor's risk aversion with interval data.

\section{Acknowledgements}

The authors would like to thank the referees for their comments and suggestions that led the paper into the current form.

\section{References}

[1] Markowitz H 1952 Portfolio selection. J. Finance 7(1): 77-91

[2] Markowitz H 1959 Portfolio selection: efficient diversification of investments. New York: Wiley

[3] Konno H and Wijayanayake A 2001 Portfolio optimization problem under concave transaction costs and minimal transaction unit constraints. Math. Program. 89(2): 233-250

[4] Kellerer H, Mansini R and Speranza M G 2000 Selecting portfolios with fixed costs and minimum transaction lots. Ann. Oper. Res. 99(1-4): 287-304

[5] Fang Y, Lai K K and Wang S Y 2006 Portfolio rebalancing model with transaction costs based on fuzzy decision theory. Eur. J. Oper. Res. 175(2): 879-893

[6] Choi U J, Jang B G and Koo H K 2007 An algorithm for optimal portfolio selection problem with transaction costs and random lifetimes. Appl. Math. Comput. 191(1): 239-252
[7] Glen J J 2011 Mean-variance portfolio rebalancing with transaction costs and funding changes. J. Oper. Res. Soc. 62(4): 667-676

[8] Zhang W G, Zhang X and Chen Y 2011 Portfolio adjusting optimization with added assets and transaction costs based on credibility measures. Insur. Math. Econ. 49(3): 353-360

[9] Zhang X, Zhang W G and Cai R 2010 Portfolio adjusting optimization under credibility measures. J. Comput. Appl. Math. 234(5): 1458-1465

[10] Woodside-Oriakhi M, Lucas C and Beasley J 2013 Portfolio rebalancing with an investment horizon and transaction costs. Omega 41(2): 406-420

[11] Mao J C 1970 Models of capital budgeting, EV vs ES. J. Finan. Quant. Anal. 4(05): 657-675

[12] Konno H and Yamazaki H 1991 Mean-absolute deviation portfolio optimization model and its applications to Tokyo stock market. Manage. Sci. 37(5): 519-531

[13] Simaan Y 1997 Estimation risk in portfolio selection: the mean variance model versus the mean absolute deviation model. Manage. Sci. 43(10): 1437-1446

[14] Chunhachinda P, Dandapani K, Hamid S and Prakash A J 1997 Portfolio selection and skewness: evidence from international stock markets. J. Bank. Finance 21(2): $143-167$

[15] Konno H and Suzuki K I 1995 A mean-variance-skewness portfolio optimization model. J. Oper. Res. Soc. Jpn. 38(2): 173-187

[16] Konno H and Shirakawa H 1994 Equilibrium relations in a capital asset market: a mean absolute deviation approach. Finance Eng. Jpn. Markets 1(1): 21-35

[17] Grootveld H and Hallerbach W 1999 Variance vs downside risk: is there really that much difference? Eur. J. Oper. Res. 114(2): 304-319

[18] Jansen D W, Koedijk K G and de Vries C G 2000 Portfolio selection with limited downside risk. J. Empirical Finance 7(1): 247-269 (special issue on Risk Management)

[19] Yang J and Qiu W 2005 A measure of risk and a decisionmaking model based on expected utility and entropy. Eur. J. Oper. Res. 164(3): 792-799

[20] Young M R 1998 A minimax portfolio selection rule with linear programming solution. Manag. Sci. 44(5): 673-683

[21] Cai X, Teo K L, Yang X and Zhou X Y 2000 Portfolio optimization under a minimax rule. Manag. Sci. 46(7): 957-972

[22] Teo K and Yang X 2001 Portfolio selection problem with minimax type risk function. Ann. Oper. Res. 101(1-4): 333-349

[23] Deng X T, Li Z F and Wang S Y 2005 A minimax portfolio selection strategy with equilibrium. Eur. J. Oper. Res. 166(1): 278-292

[24] Wu Z W, Song X F, Xu Y Y and Liu K 2009 A note on a minimax rule for portfolio selection and equilibrium price system. Appl. Math. Comput. 208(1): 49-57

[25] Sharma A and Mehra A 2013 Portfolio selection with a minimax measure in safety constraint. Optimization 62(11): 1473-1500

[26] Ida M 2003 Portfolio selection problem with interval coefficients. Appl. Math. Lett. 16: 709-713

[27] Lai K, Wang S, Xu J, Zhu S and Fang Y 2002 A class of linear interval programming problems and its application to portfolio selection. IIEE Trans. Fuzzy Syst. 10: 698-704 
[28] Giove S, Funari S and Nardelli C 2006 An interval portfolio selection problem based on regret function. Eur. J. Oper. Res. 170(1): 253-264

[29] Liu S T 2011 The mean-absolute deviation portfolio selection problem with interval-valued returns. J. Comput. Appl. Math. 235: 4149-4157

[30] Tan M 2012 Interval number model for portfolio selection with liquidity constraints. In: Fuzzy engineering and operations research, vol. 147. Berlin-Heidelberg: Springer, pp. 31-39

[31] Liu Y J, Zhang W G and Zhang P 2013 A multi-period portfolio selection optimization model by using interval analysis. Econ. Model. 33(0): 113-119

[32] Wu M, Kong D W, Xu J P and Huang N J 2013 On interval portfolio selection problem. Fuzzy Optim. Decis. Ma. 12(3): 289-304

[33] Kumar P, Panda G and Gupta U C 2015 Portfolio rebalancing model with transaction costs using interval optimization. Opsearch 52(4): 827-860
[34] Kumar P, Panda G and Gupta U C 2013 Generalized quadratic programming problem with interval uncertainty. In: Proceedings of the 2013 IEEE International Conference on Fuzzy Systems (FUZZ), pp. 1-7

[35] Kumar P, Panda G and Gupta U C 2016 An interval linear programming approach for portfolio selection model. Int. J. Oper. Res. 27(1-2): 149-164

[36] Bhurjee A K, Kumar P and Panda G 2015 Optimal range of Sharpe ratio of a portfolio model with interval parameters. $J$. Inf. Optim. Sci. 36(4): 367-384

[37] Markov S 1979 Calculus for interval functions of a real variable. Computing 22(4): 325-337

[38] Yan X S 2008 Liquidity, investment style, and the relation between fund size and fund performance. J. Financ. Quant. Anal. 43: 741-767

[39] Kumar P and Panda G 2017 Solving nonlinear interval optimization problem using stochastic programming technique. Opsearch 54(4): 752-765 